\title{
Xiaokeping mixture inhibits diabetic nephropathy in streptozotocin-induced rats through blocking TGF- $\beta$ I/Smad7 signaling
}

This article was published in the following Dove Press journal:

Drug Design, Development and Therapy

30 November 2015

Number of times this article has been viewed

\section{Chuanwei Xin \\ Zhongni Xia \\ Cheng Jiang \\ Mengmeng Lin \\ Gonghua Li}

Department of Pharmacy, Zhejiang Academy of Traditional Chinese Medicine, Tongde Hospital of Zhejiang Province, Hangzhou, People's Republic of China
Correspondence: Gonghua Li Department of Pharmacy, Zhejiang Academy of Traditional Chinese Medicine, Tongde Hospital of Zhejiang Province, Gucui Road, 310020 Hangzhou, People's Republic of China Tel +86 57I 89972240

Email xyaoshi9@163.com
Background: Diabetic nephropathy (DN) is a major cause of chronic kidney failure and characterized by excessive deposition of extracellular matrix. Evidence have shown that transforming growth factor- $\beta 1$ (TGF- $\beta 1$ ) is a key mediator in the development of DN. However, treatment of $\mathrm{DN}$ by blocking the TGF- $\beta 1 / \mathrm{Smad} 7$ pathway remains limited. Xiaokeping mixture (XKP), a traditional Chinese herbal compound, has been used for treatment in patients with DN for many years.

Methods: In the present study, TGF- $\beta 1 / \mathrm{Smad} 7$ pathway analysis was used to evaluate the therapeutic effect of XKP on DN rats induced by streptozotocin and to address the underlying molecular mechanism. Male rats were divided into four groups: normal control, untreated control group (fed with high fat), irbesartan-treated DN, and XKP-treated DN, respectively. Levels of serum creatinine, blood urea nitrogen, urine protein of 24 hours, and triacylglycerol were detected. Pathological changes of renal tissues were observed by hematoxylin-eosin staining. Immunohistochemical and Western blot analysis were used to detect the expressions of TGF- $\beta 1$ and Smad7.

Results: The results demonstrated that XKP can effectively reduce the levels of glucose, serum creatinine, blood urea nitrogen, urine protein of 24 hours, and triacylglycerol. Further studies indicated that inhibition of DN in XKP-treated DN rats was associated with inhibition of TGF- $\beta 1 /$ Smad7 signaling as demonstrated by downregulation of TGF- $\beta 1$ but upregulation of Smad7.

Conclusion: The data obtained from the present study indicate that XKP may be a therapeutic agent for DN.

Keywords: Xiaokeping mixture, diabetic nephropathy, transforming growth factor-beta, Smad7

\section{Introduction}

Diabetic nephropathy (DN) is a major cause of chronic kidney failure and characterized by excessive deposition of extracellular matrix (ECM). ${ }^{1}$ Persistent ECM production can be accelerated with severe renal injury, which results in large amounts of fibrinous tissue are generated and thus a vicious circle is formed progressively. ${ }^{2,3}$ So, it is essential to identify appropriate pharmacologic interventions to prevent renal tubulointerstitial fibrosis, especially to improve recovery of ECM following renal injury. Transforming growth factor- $\beta 1$ (TGF- $\beta 1$ ) signaling is a well-recognized pathway leading to the development of DN. ${ }^{4}$ A typical role of TGF- $\beta 1$ is its biologic effects can exert through the Smad protein signaling pathways. Thus, inhibiting the TGF- $\beta 1 /$ Smad signaling pathway is helpful for preventing renal tubulointerstitial fibrosis and preserving renal function. ${ }^{5}$ 
For thousands of years, traditional chinese medicines (TCMs) have played an important role in health maintenance for peoples throughout the world. Xiaokeping mixture (XKP) is a TCM preparation developed from a famous TCM doctor, Mr Kuijun Shi (approved by the Food and Drug Administration of Zhejiang province, medical license H20100002). It composed of Astragalus membranaceus, Rhizoma dioscoreae, Radix rehmanniae preparata, Radix ophiopogonis, radices trichosanthis, and chrysanthemum. XKP has been used for the treatment of diabetic mellitus for many years. Our previous studies have shown that XKP could decrease fasting blood glucose levels, increase insulin sensitivity index, etc. ${ }^{6,7}$ However, as TCMs usually operate in vivo through multi-components, multi-ways, and multitargets, the molecular mechanisms of XKP remain unclear. In the present study, we sought to determine whether XKP has therapeutic potential for $\mathrm{DN}$ and investigated underlying mechanisms of its action in rats with accelerated diabetic kidney.

\section{Methods}

\section{Animal and experimental protocols}

All experimental procedures were conducted in conformity with the ethics committee of Tongde Hospital of Zhejiang province, and in compliance with the National Institutes of Health Guide for the Care and Use of Laboratory Animals. Male Sprague-Dawley rats with weights ranging from 180 to $220 \mathrm{~g}$ were purchased from the Shanghai SLAC Laboratory Animal CO. LTD. The rats were housed in an air conditioned room at $24^{\circ} \mathrm{C}-25^{\circ} \mathrm{C}$, humidity of $65 \%-69 \%$ under a 12-hour dark/light cycle, and were given food and water freely. After 1 week adaptation, the rats were divided into a normal control group ( $\mathrm{NC}, \mathrm{n}=12$ ) that was fed a standard diet and a high-fat (HF) group that received HF diet (containing $67.5 \%$ standard laboratory rat chow, 15\% lard, 15\% sugar, $2 \%$ cholesterol, and $0.5 \%$ bile salts). After 4 weeks, rats on the HF diet were treated with a single intravenous injection of $60 \mathrm{mg} / \mathrm{kg}$ streptozotocin (Sigma-Aldrich, Inc., St Louis, MO, USA). Animals were considered to be diabetic if they had plasma glucose concentrations of $16.7 \mathrm{mmol} / \mathrm{L}$ or greater, in addition to polyuria and other diabetic features. All rats were randomly divided into three groups as follows ( $\mathrm{n}=12$ each group): (1) untreated control group (fed with $\mathrm{HF}$, HF group); (2) irbesartan-treated group (an ARB as known positive control, irbesartan-treated DN [IRB-DN]; $17.5 \mathrm{mg} / \mathrm{kg} /$ day, diluted in $0.5 \%$ carboxymethyl cellulose); and (3) XKP-treated DN (XKP-DN, $1.2 \mathrm{~g} / \mathrm{kg} /$ day, diluted in $0.5 \%$ carboxymethyl cellulose). All drugs were administered via intra-gastric gavage once a day for 16 weeks.

\section{Biochemical analysis and light microscopy}

Body weight of rats was measured after treatments for 16 weeks, and blood of rats was sampled from the tail vein, the blood glucose levels were measured by a One Touch blood glucose monitoring system. And metabolic cages were used to collect urine samples of 24 hours, the urinary protein excretion was determined by the Bradford method. The total cholesterol, triglycerides, urea nitrogen, and serum creatinine were detected by an automatic biochemistry analyzer. Tissue for light microscopy was fixed in $4 \%(w / v)$ paraformaldehyde and then embedded in paraffin. Four micrometer thick sections of renal tissue were processed for hematoxylin-eosin and Masson's trichrome staining.

\section{Western blot analysis}

Renal proteins were fractionated by sodium dodecyl sulfate polyacrylamide gelelectrophoresis (SDS-PAGE) and transferred onto nitrocellulose difluoride membranes. The membranes were blocked in $5 \%$ bovine serum albumin for 1 hour and incubated with a primary antibody at $4{ }^{\circ} \mathrm{C}$ overnight, then they were washed in Tris-buffered saline, $0.1 \%$ Tween 20 , and incubated with secondary antibody at $20^{\circ} \mathrm{C}$ for 1 hour. After washing, signals were visualized with an enhanced chemiluminescent system. Protein levels were normalized against $\beta$-actin.

\section{Statistical analysis}

The data were presented as the mean \pm standard deviation. Statistical analysis was carried out using SPSS 19.0. The differences among multiple groups were evaluated by one-way analysis of variances. $P<0.05$ was considered as statistically significant.

\section{Results}

\section{Animal characteristics}

At the beginning of study, all of the rats behaved normally. During the experiment, the rats in the HF diet group gradually showed listlessness, polyuria, and lost weight. After 7 weeks, the rats in the HF group appeared symptoms such as ulcer and cataracts. After 16 weeks of drug therapy, the symptoms mentioned above improved significantly both in IRB-DN group and in XKP-DN group.

\section{Effects of XKP on bodyweight, blood glucose, and 24 hours urinary protein}

At the end of study, two rats died in the HF group and all rats were alive in the other groups. After 16 weeks of drug therapy, rats in NC group displayed progressive gain of body weight whereas rats in HF group showed less gain of body weight. However, either in XKP-DN group or in IRB-DN group the 
Table I Effect of XKP on weight/kidney index in rats with DN at 16 th week

\begin{tabular}{lll}
\hline Group & $\begin{array}{l}\text { Body } \\
\text { weight }(\mathrm{g})\end{array}$ & $\begin{array}{l}\text { Kidney/body } \\
\text { weight (\%) }\end{array}$ \\
\hline NC & $492.0 \pm 39.7$ & $0.757 \pm 0.02$ \\
HF & $204.9 \pm 47.7^{*}$ & $1.89 \pm 0.36^{*}$ \\
IRB-DN & $284.2 \pm 28.7^{*, \Delta}$ & $1.35 \pm 0.32^{*, \Delta}$ \\
XKP-DN & $285.4 \pm 29.1^{*, \Delta}$ & $1.29 \pm 0.35^{*, \Delta}$ \\
\hline
\end{tabular}

Notes: $* P<0.01$ compared with $N C$ rats and $\triangle P<0.05$ compared with HF rats. Data shown as mean \pm standard deviation.

Abbreviations: DN, diabetic nephropathy; HF, high fat; IRB, irbesartan; IRB-DN, IRB-treated DN; NC, normal control; XKP, Xiaokeping mixture; XKP-DN, XKPtreated DN.

drug did not prevent the reduction in body weight, as shown in Table 1. Rats with untreated DN showed severe hyperglycemia characterized by elevated fasting and postprandial plasma glucose levels $(P<0.01)$. However, treatment with XKP decreased plasma glucose levels significantly, when compared with those of HF group rats $(P<0.05)$ (Table 2). The blood urea nitrogen, serum creatinine, and urinary total protein increased significantly in comparison with those of $\mathrm{NC}$ group rats $(P<0.01)$. However, treatment with both XKP and irbesartan reduced the ratio of kidney to body weight and reversed renal dysfunction and proteinuria $(P<0.05)$.

As shown in Table 3, rats with untreated DN showed severe dyslipidemia. The serum total cholesterol, triglycerides, and low-density lipoprotein cholesterol; levels have increased significantly, when compared with those of $\mathrm{NC}$ group rats $(P<0.01)$. However, treatment with XKP and irbesartan alleviated hyperlipidemia significantly in rats with $\mathrm{DN}(P<0.05)$.

\section{Effects of XKP on renal structure}

As shown in Figure 1, glomerular and tubular structures were examined by periodic acid-Schiff staining, there were no obvious abnormalities in glomerular and tubular structures of the kidneys in rats of $\mathrm{NC}$ group. However, there was a significant mesangial expansion, thickened basement membranes, and occluded capillaries in rats of HF group,

Table 2 Effect of XKP on GLU and renal function in rats with DN at 16th week

\begin{tabular}{lllll}
\hline Group & $\begin{array}{l}\text { GLU } \\
(\mathbf{m m o l} / \mathbf{L})\end{array}$ & $\begin{array}{l}\text { BUN } \\
(\mathbf{m m o l} / \mathrm{L})\end{array}$ & $\begin{array}{l}\mathbf{S C r} \\
(\mu \mathrm{mol} / \mathrm{L})\end{array}$ & $\begin{array}{l}\text { Urinary } \\
\text { total protein } \\
\mathbf{( m g / 2 4} \mathbf{~ h})\end{array}$ \\
\hline $\mathrm{NC}$ & $5.20 \pm 3.69$ & $5.57 \pm 0.82$ & $56.59 \pm 1.22$ & $18.25 \pm 2.28$ \\
$\mathrm{HF}$ & $24.33 \pm 5.04^{*}$ & $15.02 \pm 1.32^{*}$ & $92.5 \mathrm{I} \pm 1.15^{*}$ & $92.24 \pm 2.46^{*}$ \\
$\mathrm{IRB}-\mathrm{NN}$ & $11.44 \pm 6.35^{*, \Delta}$ & $8.24 \pm 0.79^{*, \Delta}$ & $78.63 \pm 1.94^{*, \Delta}$ & $52.82 \pm 2.8 \mathrm{I}^{*, \Delta}$ \\
XKP-DN & $9.82 \pm 4.24^{*, \Delta}$ & $8.32 \pm 0.8 \mathrm{I}^{*, \Delta}$ & $80.44 \pm 1.85^{*, \Delta}$ & $55.09 \pm 3.07^{*, \Delta}$ \\
\hline
\end{tabular}

Notes: $* P<0.01$ compared with $\mathrm{NC}$ rats and $\Delta \mathrm{P}<0.05$ compared with HF rats. Data shown as mean \pm standard deviation.

Abbreviations: BUN, blood urea nitrogen; DN, diabetic nephropathy; GLU, glucose; $\mathrm{HF}$, high fat; IRB-DN, irbesartan-treated DN; NC, normal control; SCr, serum creatinine; XKP, Xiaokeping mixture; XKP-DN, XKP-treated DN; h, hours.
Table 3 Effect of XKP on plasma lipid profiles in rats with DN at 16th week

\begin{tabular}{lllll}
\hline Group & $\begin{array}{l}\text { TG } \\
(\mathbf{m m o l} / \mathbf{L})\end{array}$ & $\begin{array}{l}\text { TC } \\
(\mathbf{m m o l} / \mathbf{L})\end{array}$ & $\begin{array}{l}\text { LDL-C } \\
\mathbf{( m m o l} / \mathbf{L})\end{array}$ & $\begin{array}{l}\text { HDL-C } \\
\mathbf{( m m o l} / \mathbf{L})\end{array}$ \\
\hline NC & $1.14 \pm 0.22$ & $2.07 \pm 0.28$ & $0.59 \pm 0.15$ & $1.55 \pm 0.28$ \\
HF & $2.82 \pm 0.55^{*}$ & $7.92 \pm 1.42^{*}$ & $4.64 \pm 1.55^{*}$ & $1.24 \pm 0.22$ \\
IRB-DN & $1.73 \pm 0.42^{*, \Delta}$ & $5.24 \pm 0.92^{*, \Delta}$ & $1.73 \pm 0.27^{*, \Delta}$ & $1.47 \pm 0.32$ \\
XKP-DN & $1.74 \pm 0.46^{*, \Delta}$ & $4.92 \pm 0.84^{*, \Delta}$ & $1.74 \pm 0.28^{*, \Delta}$ & $1.49 \pm 0.35$ \\
\hline
\end{tabular}

Notes: $* P<0.01$ compared with NC rats and $\triangle P<0.05$ compared with HF rats. Data shown as mean \pm standard deviation.

Abbreviations: DN, diabetic nephropathy; HDL-C, high-density lipoprotein cholesterol; $\mathrm{HF}$, high fat; IRB-DN, irbesartan-treated DN; LDL-C, low-density lipoprotein cholesterol; NC, normal control; XKP, Xiaokeping mixture; XKP-DN, XKP-treated DN; TC, total cholesterol; TG, triglycerides.

and there was a significant collagen deposition and fibrosis in the tubulo-interstitium of rats. However, both XKP and irbesartan significantly reduced the changes in both glomerular and tubular structures.

\section{Effects of XKP on TGF- $\beta$ I and Smad7 expression}

As shown in Figure 2, there were positive particles of TGF- $\beta 1$ protein expression in rats of NC group (Figure 2A), with a little of $\tan$ grain. Whereas there were a lot of $\tan$ particles in rats of HF group (Figure 2B, compare with Figure 2A, $P<0.01)$. Compare with HF group, there were much little particles in IRB-DN group (Figure $2 \mathrm{C}, P<0.05$ ) or in XKP-DN group (Figure $2 \mathrm{D}, P<0.05$ ), suggesting that TGF- $\beta 1$ protein expression have increased on the DN rat kidney tissue; however, both XKP and irbesartan significantly reduced the TGF- $\beta 1$ protein expression on the DN rat kidney tissue.

In the cytoplasm, there were positive of Smad7 protein expression in rats of $\mathrm{NC}$ group (Figure 2E), with a lot of tan particles, and the color is deep. Whereas there were much little tan particles in rats of HF group (Figure $2 \mathrm{~F}$, compare with Figure 2E, $P<0.01)$. Compare with HF group, there were much more tan particles in IRB-DN group (Figure $2 \mathrm{G}, P<0.05$ ) or in XKP-DN group (Figure $2 \mathrm{H}, P<0.05$ ), suggesting that Smad7 protein expression have reduced on the $\mathrm{DN}$ rat kidney tissue. However, both XKP and irbesartan significantly increased the Smad7 protein expression on the DN rat kidney tissue.

We next investigated the mechanisms by which XKP treatment attenuated $\mathrm{DN}$ by studying the TGF- $\beta 1 / \mathrm{Smad} 7$ signaling pathway. TGF- $\beta 1 / \mathrm{Smad} 7$ signaling was highly activated in the diabetic kidney as revealed by a marked upregulation of TGF- $\beta 1$, but lower levels of an inhibitory Smad7 protein expression level, as shown in Figure 3. Treatment with XKP significantly reduced the TGF- $\beta 1$ protein expression on the DN rat kidney tissue, which was associated with significantly increased Smad7 protein expression. 

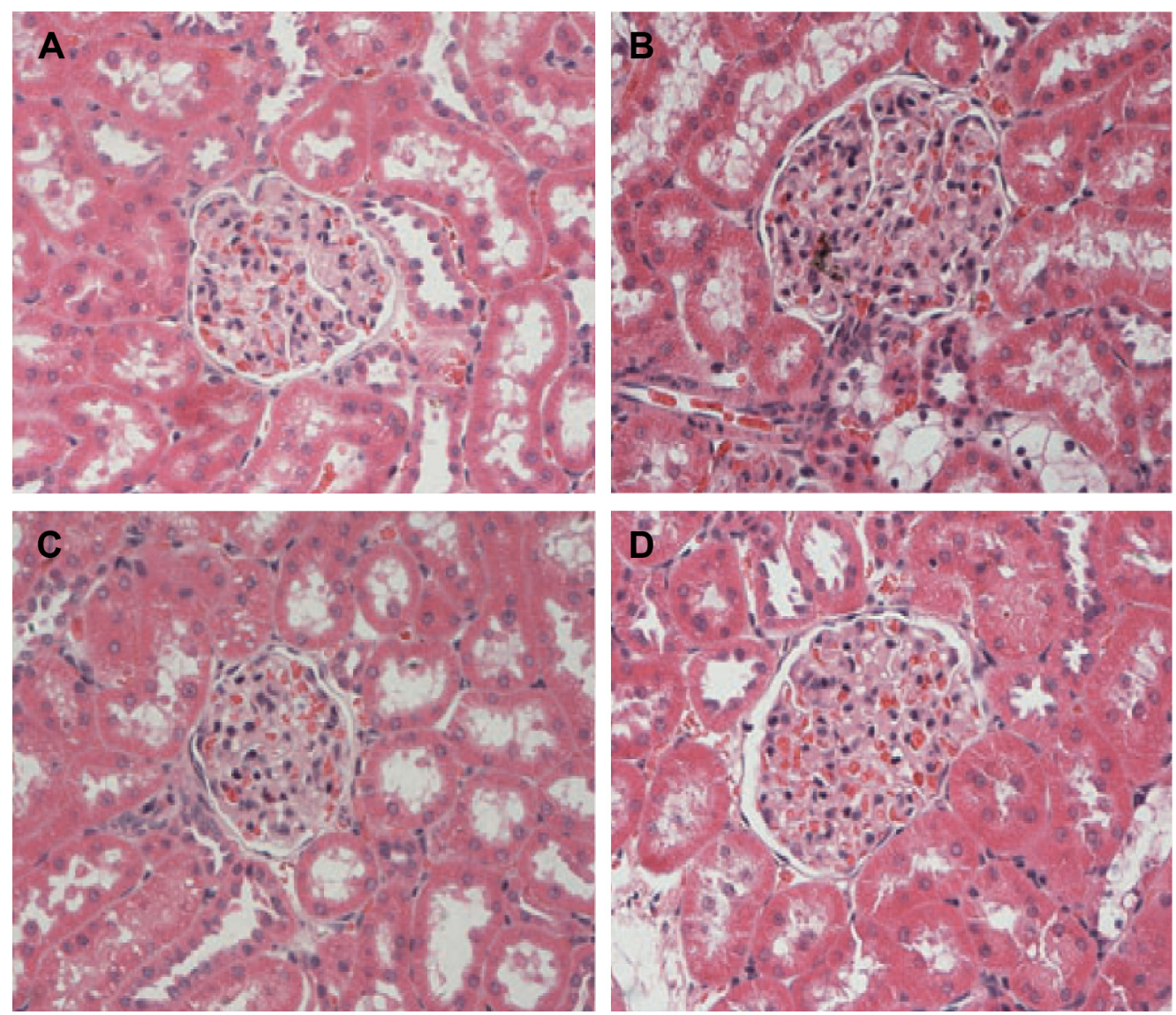

Figure I Effects of XKP on renal pathology of experimental rats.

Notes: Representative pictures of PAS staining (original magnification $\times 400$ ) are presented as (A-D). (A) NC group; (B) HF group; (C) IRB-DN; and (D) XKP-DN.

Abbreviations: DN, diabetic nephropathy; HF, high fat; IRB-DN, irbesartan-treated DN; NC, normal control; PAS, periodic acid-Schiff; XKP, Xiaokeping mixture; XKPDN, XKP-treated DN.
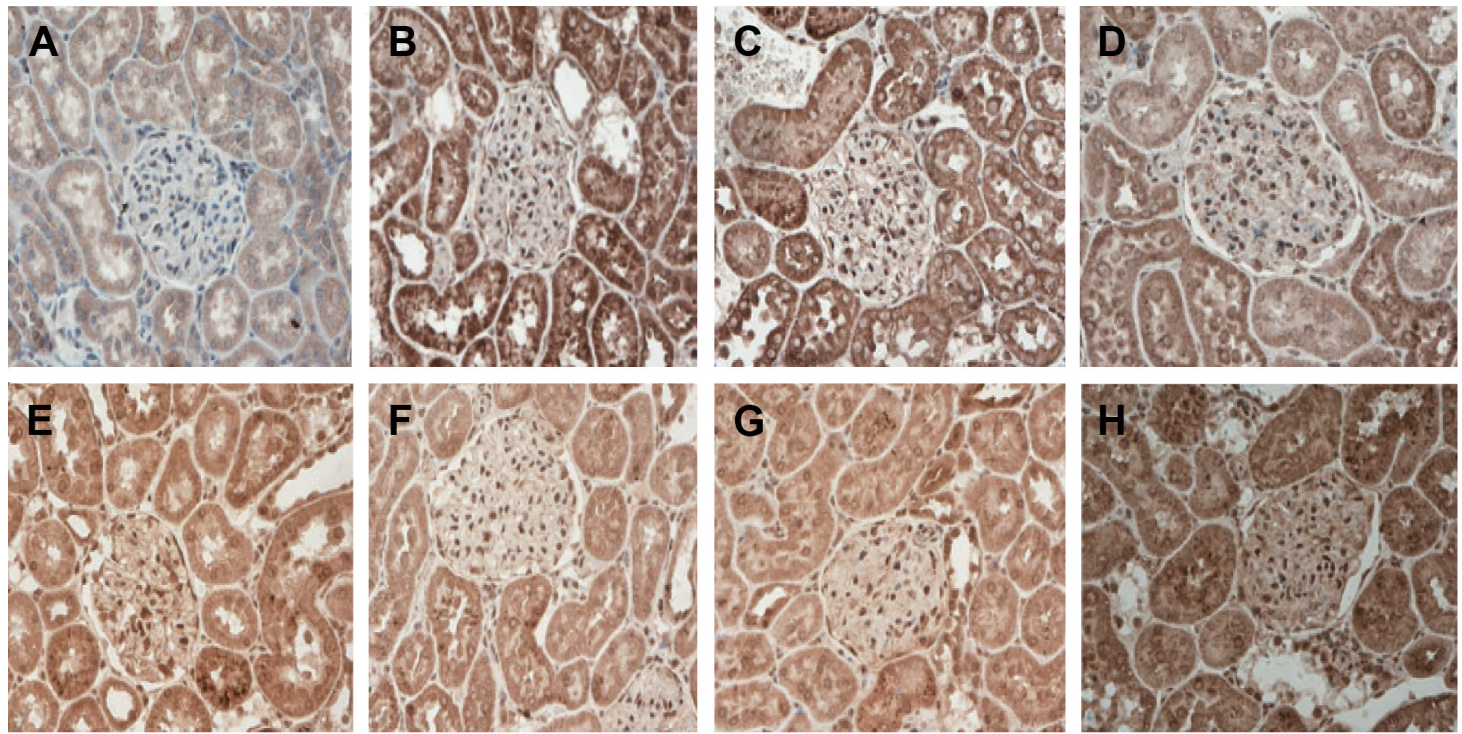

Figure 2 Effects of XKP on TGF- $\beta$ I and Smad7 expression of experimental rats.

Notes: Representative pictures of PAS staining (original magnification $\times 400$ ) are presented as $(\mathbf{A}-\mathbf{H})$. (A and $\mathbf{E})$ NC group; (B and F) HF group; (C and $\mathbf{G})$ IRB-DN; and (D and $\mathbf{H})$ XKP-DN.

Abbreviations: DN, diabetic nephropathy; HF, high fat; IRB-DN, irbesartan-treated DN; NC, normal control; PAS, periodic acid-Schiff; TGF- $\beta$ I, transforming growth factor-BI; XKP, Xiaokeping mixture; XKP-DN, XKP-treated DN. 


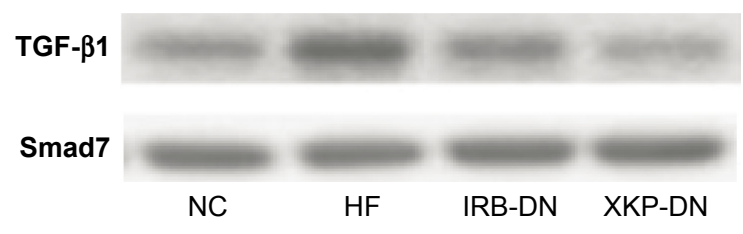

Figure 3 Western blot analysis of XKP on TGF- $\beta$ I and Smad7 expression. Abbreviations: DN, diabetic nephropathy; HF, high fat; IRB-DN, irbesartantreated DN; NC, normal control; TGF- $\beta$ I, transforming growth factor- $\beta$ I; XKP Xiaokeping mixture; XKP-DN, XKP-treated DN.

\section{Discussion}

TCM formulas are an important source for the development of new drugs to prevent and treat diabetes and its complications. ${ }^{8}$ In the present study, we examined the therapeutic effect and the underlying mechanisms of XKP in a rat model of DN. We found that administration of XKP significantly inhibited streptozotocin-induced kidney fibrosis and markedly decreased proteinuria. The inhibitory effect of XKP on diabetic kidney disease was associated with inactivation of TGF- $\beta 1 /$ Smad 7 signaling.

$\mathrm{DN}$ is characterized by pathophysiological changes in glomerular hyperfiltration, renal hypertrophy, tubular function and then progress to proteinuria and reduction in glomerular filtration rate. ${ }^{9}$ Many studies have demonstrated that TGF- $\beta 1$ plays an important role in experimental diabetic kidney disease and human DN. ${ }^{10,11}$ TGF- $\beta 1$ is a key profibrotic cytokine that acts by stimulating synthesis of ECM components, decreasing matrix degradation, and promoting cell-matrix interactions. Moreover, TGF- $\beta 1$ is also involved in tubule glomerular sclerosis and podocyte apoptosis in diabetes. Smad7, an inhibitory Smad, can block TGF- $\beta 1 /$ Smad signaling by binding to Smurf2 to form an E3 ubiquitin ligase that targets the TGF- $\beta 1$ receptor as well as Smads including Smad7 for degradation. ${ }^{12}$ Once Smad7 is degraded, activation of Smad3 and renal fibrosis is enhanced. The functional importance of TGF- $\beta 1 / \mathrm{Smad}$ signaling in DN is demonstrated in a number of animal models, in which overexpression of Smad7 inhibits diabetic renal injury. ${ }^{13-15}$ In our present study, treatment with XKP attenuated diabetic renal injury by rebalancing the TGF- $\beta 1 /$ Smad signaling pathway. A similar mechanism was also evident by a positive treatment control with irbesartan. Thus, inhibition of TGF- $\beta 1 / \mathrm{Smad} 7$ signaling could be an important mechanism, by which XKP attenuated diabetic kidney disease.

Practitioners of TCM believe that in the early stage of DN, patients mostly exhibit deficiency in conjunction with dryness-hest in lung and spleen. This was manifested by frequent urination and easily getting thirsty, soreness and weakness of waist and knees, dizziness, and tinnitus.
During the course of DN, the yin deficiency spreads to the yang, causing a yin-and-yang deficiency. XKP has long been used for the treatment of "kidney-Yang" deficiency syndrome. Accumulating evidence from the ethnopharmacological aspect indicates that the herbs and their chemical constituents are beneficial for the recovery of renal function, for example, A. membranaceus, also known as Huangqi in People's Republic of China, has been frequently used in TCM for several centuries. As one of the 50 fundamental herbs, it is traditionally employed for the treatment of diabetes, for wound healing and strengthening immunity. ${ }^{16}$ It also possesses anti-inflammatory, hepatoprotective, antioxidative, and cardioprotective properties. ${ }^{17,18}$

\section{Conclusion}

In the present study, we performed an analysis to evaluate the therapeutic effect of XKP treatment on DN rats and address the underlying molecular mechanism. Our results indicate that XKP treatment could ameliorate DN symptoms and inhibiting glucose and lipid metabolism. The mechanism is closely related to inhibit TGF- $\beta 1 /$ Smad7 signaling, which further underscoring the potential clinical benefits of XKP in the treatment of DN.

\section{Acknowledgments}

We would like to acknowledge Dr Yun-Long Tian (research question development, study selection, and interpretation of results) and Mrs Ting-Xia (data analysis and interpretation of results) for their involvement. This study was supported by Zhejiang Provincial Natural Science Foundation of China (LY14H280003).

\section{Disclosure}

The authors report no conflicts of interest in this work.

\section{References}

1. Gao LH, Liu Q, Liu SN, et al. A refined-JinQi-JiangTang tablet ameliorates prediabetes by reducing insulin resistance and improving beta cell function in mice. J Ethnopharmacol. 2014;151(1):675-685.

2. Forbes JM, Cooper ME. Mechanisms of diabetic complications. Physiol Rev. 2013;93(1):137-188.

3. Maezawa Y, Takemoto M, Yokote K. Cell biology of diabetic nephropathy: roles of endothelial cells, tubulointerstitial cells and podocytes. J Diabetes Investig. 2015;6(1):3-15.

4. Cooper ME. Diabetes: treating diabetic nephropathy - still an unresolved issue. Nat Rev Endocrinol. 2012;8(9):515-516.

5. Hills CE, Squires PE. The role of TGF- $\beta$ and epithelial-to mesenchymal transition in diabetic nephropathy. Cytokine Growth Factor Rev. 2011;22(3):131-139.

6. Xin CW, Huang P, Chen XW, et al. Observation on improvement of islet $\beta$-cell function in recent-onset type 2 diabetes by Xiaokeping mixture. Chin J Mod Appl Pharm. 2011;28(1):81-84. 
7. Xin CW, Huang P, Tian YL, et al. Research of xiaokeping mixture on vascular endothelial growth factor in early diabetic nephropathy patients. Chin Arch Trad Chin Med. 2014;32(7):560-561.

8. Junhua Z, Hongcai S, Xiumei G, et al. Methodology and reporting quality of systematic review/meta-analysis of traditional Chinese medicine. J Altern Complement Med. 2007;13(8):797-805.

9. Liu X, Liu L, Chen P, et al. Clinical trials of traditional Chinese medicine in the treatment of diabetic nephropathy - A systematic review based on a subgroup analysis. J Ethnopharmacol. 2014;151(2):810-819.

10. Abdel-Rahman EM, Saadulla L, Reeves WB, et al. Therapeutic modalities in diabetic nephropathy: standard and emerging approaches. J Gen Intern Med. 2012;27(4):458-468.

11. McGowan TA, Yanqing Zhu Y, Sharma K. Transforming growth factorbeta: a clinical target for the treatment of diabetic nephropathy. Curr Diab Rep. 2004;4(6):447-454.

12. Lan HY. Transforming growth factor- $\beta /$ Smad signalling in diabetic nephropathy. Clin Exp Pharmacol Physiol. 2012;39(8):731-738.

13. Wu JS, Liu Y, Shi R, et al. Effects of combinations of Xie xin decoction constituents on diabetic nephropathy in rats. $J$ Ethnopharmacol. 2014;157:126-133.
14. Abe HM, Atsubara T, Arai H, Doi T. Role of Smad1 in diabetic nephropathy: Molecular mechanisms and implications as a diagnostic marker. Histol Histopathol. 2011;26(4):531-541.

15. Ishibe S, Cantley LG. Epithelial-mesenchymal-epithelial cycling in kidney repair. Curr Opin Nephrol Hypertens. 2008;17(4):379-385.

16. Xie W, Zhang Y, Wang N, et al. Novel effects of macrostemonoside A, a compound from Allium macrostemon Bung, on hyperglycemia, hyperlipidemia, and visceral obesity in high-fat diet-fed C57BL/6 mice. Eur J Pharmacol. 2008;599(1-3):159-165.

17. Yan Y, Feng Y, Li W, et al. Protective effects of quercetin and hyperoside on renal fibrosis in rats with unilateral ureteral obstruction. Exp Ther Med. 2014;8(3):727-730.

18. Wang B, Jha JC, Hagiwara S, et al. Transforming growth factor- $\beta 1$ mediated renal fibrosis is dependent on the regulation of transforming growth factor receptor 1 expression by let-7b. Kidney Int. 2014; 85(2):352-361.

\section{Publish your work in this journal}

Drug Design, Development and Therapy is an international, peerreviewed open-access journal that spans the spectrum of drug design and development through to clinical applications. Clinical outcomes, patient safety, and programs for the development and effective, safe, and sustained use of medicines are a feature of the journal, which has also been accepted for indexing on PubMed Central. The manuscript management system is completely online and includes a very quick and fair peer-review system, which is all easy to use. Visit http://www.dovepress.com/testimonials.php to read real quotes from published authors.

Submit your manuscript here: http://www.dovepress.com/drug-design-development-and-therapy-journal 